\title{
Does plant growth accelerate silicate weathering?
}

\author{
FRIEDHELM VON BLANCKENBURG ${ }^{1}$ AND DR. RALF A. \\ OESER $^{2}$
}

${ }^{1}$ GFZ German Research Centre for Geosciences

${ }^{2}$ GFZ Potsdam

Presenting Author: ralf.oeser@icloud.com

Plants and their associated below-ground micro-biota are thought to enhance silicate weathering by increasing soil $\mathrm{CO}_{2}$, excreting organic ligands that solubilise elements, nutrient uptake by deep roots, and by associated fungi directly enhancing primary mineral dissolution. Weathering fluxes thus could be, and often are, hypothesised to increase with plant growth. As test of this hypothesis we quantified the degree and rate of weathering and compared these to nutrient uptake into the principle higher plants along the "EarthShape" transect in the Chilean Coastal Cordillera. These granitoid sites define a dramatic gradient featuring a five-fold increase in precipitation and net primary productivity (NPP) from north to south. We found that neither the degree of weathering nor the weathering rates increase systematically with precipitation or biomass growth along the climate and vegetation gradient. Instead, the increase in nutrient demand with increasing biomass growth is accommodated by faster nutrient recycling between plants and soil. In the absence of an increase in weathering rate despite the massive increase in precipitation, we hypothesize that plant growth might in fact dampen weathering rates. If confirmed by further evidence, a more nuanced role of plants as key players in the global silicate weathering-carbon feedback has to be defined.

Oeser, R. A., \& von Blanckenburg, F. (2020). Do degree and rate of silicate weathering depend on plant productivity? Biogeosciences, doi:10.5194/bg-17-4883-2020 